\title{
Seebeck Effects and Electronic Thermal Conductivity of IV-VI Materials
}

\author{
Akihiro Ishida, Tomohiro Yamada, Takayuki NaKano, \\ Yasushi TAKANO, and Sadao TAKAOKA ${ }^{1}$ \\ Department of Electrical and Electronic Engineering, \\ Faculty of Engineering, Shizuoka University, Johoku 3-5-1, \\ Naka-ku, Hamamatsu 432-8561 \\ ${ }^{1}$ Department of Physics, Graduate School of Science, Osaka \\ University, Machikaneyama 1-1, Toyonaka 560-0043
}

Theoretical calculations of Seebeck coefficients and electronic thermal conductivity for general materials such as metals, semiconductors, and quantum structures are described, and dependence of the Seebeck coefficient on doping level and temperature is discussed for lead salt materials. Experimental Seebeck coefficient was measured for $\mathrm{PbS}$ films and the value agreed well with the theoretical one, indicating high thermoelectric performance comparable to PbTe. Temperature dependence of electronic thermal conductivity is also discussed for the lead salts. It is shown that minority carriers in the narrow gap semiconductor significantly affect the electronic thermal conductivity and electronic thermal conductivity at high temperature can be reduced by highly doping. 


\section{Introduction}

Developments of materials with high thermoelectric conversion efficiency are important for energy and environmental issues. Dimensionless figure of merits $Z T=S^{2} \sigma T / \kappa$ or power factor $P_{f}=S^{2} \sigma$, where $S$ is the Seebeck coefficient, $\sigma$ the electrical conductivity, and $\kappa$ the thermal conductivity, are used as indicators of the conversion efficiency. Among the parameters, thermal conductivity is an important parameter because the reduction of thermal conductivity increases the $\mathrm{ZT}$ and furthermore reduces the necessary amount of the material for device fabrication. Introduction of nanostructure is efficient to reduce the thermal conductivity. ${ }^{1-3)}$ Seebeck coefficient is another important factor because it has the effect of second power on the efficiency. Various materials and quantum structures have been reported as high efficiency thermoelectric materials, ${ }^{1-5}$ and theoretical calculations of Seebeck coefficients through band structure calculations have been also performed. In the theoretical calculation Mott formula is usually used to predict the Seebeck coefficient of metals, ${ }^{6}$ and the formula is sometimes used to discuss the Seebeck coefficients of semiconductors and quantum wells qualitatively. ${ }^{7,8}$ Recently, first principle calculations for band structures of complicated material systems and quantum wells are available owing to the developments of computers, ${ }^{9,10)}$ and these calculations are becoming important to predict high efficiency thermoelectric materials. In this paper, first, we describe theoretical calculations of Seebeck coefficients and electronic thermal conductivity for general materials such as metals, 
semiconductors, and quantum structures on the basis of solid state physics, which would be useful for both theoretical and experimental thermoelectric researchers. Next, we discuss Seebeck coefficient and electronic thermal conductivity of lead salt IV-VI materials.

\section{Theoretical Calculations of Seebeck Coefficient and Electronic Thermal Conductivity}

Figure 1 shows schematic carrier distribution on energy and carrier flows in n-type semiconductors under temperature gradient. The Seebeck coefficient is determined by the equation:

$S=(1 / e) d E_{F} / d T=(-1 / e) d\left(E_{C}-E_{F}\right) / d T+(1 / e) d E_{C} / d T$,

,where $E_{F}$ and $E_{C}$ are the Fermi level and conduction band edge energy, respectively. The first term on the right side of the equation is determined by the density of states, and the second term is determined by the balance between drift and thermal diffusion currents: $J=J_{e d}+J_{d t}+J_{h d}+J_{h t}=0$, where $J_{e d}$ and $J_{h d}$ represent drift current densities for electrons and holes, respectively, and $J_{e t}$ and $J_{h t}$ represents thermal diffusion currents for the electrons and holes, respectively. In general, the total current density is given by the equation below under the background of solid-state physics and Boltzmann equation ${ }^{11)}$

$J=\left(\frac{e^{2}}{4 \pi^{3} k T}\right) \frac{d T}{d x}\left[\sum_{V} \int \tau v_{x}^{2} f_{0}\left(1-f_{0}\right)\left(\frac{E-E_{F}}{e T}+\frac{1}{e} \frac{d E_{F}}{d T}\right) d^{3} k\right]$, 
where the summation is taken for all energy bands related to the conductions. The equation for the Seebeck coefficient $S$ is obtained from the condition $J=0$ :

$$
S=-\frac{1}{e T} \frac{\sum_{V} \int \tau v_{X}^{2} f_{0}\left(1-f_{0}\right)\left(E(\mathbf{k})-E_{F}\right) d^{3} \mathbf{k}}{\sum_{V} \int \tau v_{X}^{2} f_{0}\left(1-f_{0}\right) d^{3} \mathbf{k}},
$$

where $\tau$ is the scattering time of carrier, $V_{x}$ the electron velocity along temperature gradient, $f_{0}$ is the Fermi-Dirac distribution. In the calculation, phonon-drag effect in which phonon flow causes significant enhancement of the Seebeck coefficient at low temperature is not considered.12) After integrations over the constant energy surfaces, the equation becomes the following energy integral:

$S=-\frac{1}{e T} \frac{\sum_{V} \int \tau(E) \bar{v}_{X}^{2}(E) f_{0}\left(1-f_{0}\right)\left(E-E_{F}\right) D(E) d E}{\sum_{V} \int \tau(E) \bar{v}_{X}^{2}(E) f_{0}\left(1-f_{0}\right) D(E) d E}$,

where $D(E)$ is the density of states in the V-th valley and $\bar{v}_{X}^{2}(E)$ is the value given by

$\bar{v}_{X}^{2}(E)=\frac{1}{S_{k}} \int_{S_{k}} v_{X}^{2}(\mathbf{k}) d^{2} \mathbf{k}$.

Here, $S_{k}$ represents the area of constant energy surface with energy $E$ in the V-th valley.

For simple metal with parabolic band, Eq.(4) becomes Mott formula: 


$$
\begin{aligned}
& S_{\text {metal }} \approx-\frac{1}{e T} \frac{\int_{0}^{\infty} f_{0}\left(1-f_{0}\right)(D(E))_{E=E_{F}}\left(E-E_{F}\right)^{2} d E}{\int_{0}^{\infty} f_{0}\left(1-f_{0}\right) N(E) d E} \\
& \approx-\frac{k^{2} T}{e} \frac{D\left(E_{F}\right) \int_{-\infty}^{\infty} \frac{x^{2} e^{x}}{\left(1+e^{x}\right)^{2}} d x}{N\left(E_{F}\right) \int_{-\infty}^{\infty} \frac{e^{x}}{\left(1+e^{x}\right)^{2}} d x} \\
& =-\frac{\pi^{2} k^{2} T}{3 e}\left[\frac{\mathrm{d} \ln N(E)}{\mathrm{d} E}\right]_{E=E_{F}}=-\frac{\pi^{2} k^{2} T}{3 e}\left[\frac{\mathrm{d} \ln \sigma(E)}{\mathrm{d} E}\right]_{E=E_{F}}
\end{aligned}
$$

where we assumed $\tau$ is constant and replaced $\left(E-E_{F}\right) / k T=x$. In the calculation, we also used $\bar{v}_{X}^{2}(E) \propto E$, $D(E)=\frac{d N(E)}{d E}=3 N(E) / 2 E$, in which $N(E)$ is the number of states below $E$, and we put $N(E) \approx N\left(E_{F}\right)+(d N / d E)_{E=E_{F}}\left(E-E_{F}\right)$ and $\sigma(E)=e^{2} \tau N(E) / m$. Detailed calculation is discussed in Ref.13. In general, band structure of metals are more complicated and it is necessary to calculate the Seebeck coefficient from Eq.(4) in the case many valleys affect the conductivity.

The Mott formula is not applicable for semiconductors and superlattices. The Seebeck coefficients must be calculated from Eq.(3) or (4). In semiconductors and semimetals, constant energy surface near the Fermi energy often becomes rotational ellipsoid with longitudinal effective mass $m_{l}$ and transverse effective mass $m_{t}$, and $\bar{v}_{X}^{2}(E)$ is written as

$$
\bar{v}_{X}^{2}(E)=\frac{2}{3}\left(\frac{\cos ^{2} \phi}{m_{l}}+\frac{\sin ^{2} \phi}{m_{t}}\right) E
$$


where $E$ is measured from the band edge, and $\phi$ is the angle between the longitudinal direction and temperature gradient. In narrow gap semiconductors and semimetals, the $E^{\text {-k }}$ relationship is often represented by $E\left(1+E / E_{g}\right)=\sum \hbar^{2} k^{2} / 2 m_{i j}$ in two band flame work, and density of states $\mathrm{D}(\mathrm{E})$ and $\bar{v}_{X}^{2}(E)$ are given by

$D(E)=\frac{\sqrt{2 m_{t}^{2} m_{l} E\left(1+\frac{E}{E_{g}}\right)\left(1+\frac{2 E}{E_{g}}\right)}}{\pi^{2} \hbar^{3}}$,

and

$$
\bar{v}_{X}^{2}(E)=\frac{2}{3}\left(\frac{\cos ^{2} \phi}{m_{l}}+\frac{\sin ^{2} \phi}{m_{t}}\right) \frac{E\left(1+E / E_{g}\right)}{\left(1+2 E / E_{g}\right)^{2}} \quad .
$$

In many valley semiconductors such as lead-salt IV-VI materials, n-type Ge, or $\mathrm{n}$-type $\mathrm{Si}$, there is no directional dependence of Seebeck coefficient under no strain, because the summation below shows no directional dependence.

$$
\sum_{V}\left(\frac{\cos ^{2} \phi}{m_{l}}+\frac{\sin ^{2} \phi}{m_{t}}\right)=M\left(\frac{1}{3 m_{l}}+\frac{2}{3 m_{t}}\right) .
$$

However, if electron distribution in each valley is varied by strain, directional dependence appears.

Figure 2 shows conduction band densities of states for bulk, two-dimensional, and one-dimensinal Si. In the bulk, two-dimensional, and one-dimensional systems for the host 
materials with parabolic $E$-k relationship, the Seebeck coefficients are given by a simple equation:

$$
S=-\frac{1}{e T} \frac{\int_{0}^{\infty} f_{0}\left(1-f_{0}\right) E^{s}\left(E-E_{F}\right) d E}{\int_{0}^{\infty} f_{0}\left(1-f_{0}\right) E^{s} d E},
$$

where $\mathrm{s}$ is constant depending on the material system. Assuming $\tau(E) \propto E^{-1 / 2}$ for optical phonon scattering, and $\bar{v}_{X}^{2} \propto E, s$ becomes 1 for bulk materials, and $s=1 / 2$ for two dimensional system, and $s=0$ for one-dimensional system, depending on the densities of states for bulk, two-dimensional, and one-dimensional systems. If more than two type of carriers or quantum levels affect the conduction, summation given by Eq.(4) is necessary. Since the Seebeck coefficient is given by an average value of $\left(E_{F}-E\right) / e T$, it strongly depends on the carrier concentration, and if the carrier concentration is same, the materials with large density of states have higher Seebeck coefficient. In low dimensional systems such as superlattice, enhancement of Seebeck coefficient is possible owing to a high and step-like density of states.14) However, a high density of quantum structures is necessary to obtain enhanced Seebeck coefficient and resultant higher thermoelectric performance compared to bulk materials since the density of states $D(E)$ is proportional to the density of quantum structures. Other possibilities to enhance the Seebeck effect are filtering effects of carriers, caused at the boundary of nanostructures ${ }^{15)}$, caused by strain in many valley semiconductors and effective mass modulation in 
superlattice. ${ }^{16)}$ In this calculation we did not consider phonon drag effect, which increases the Seebeck coefficient of the material with long phonon mean free path such as Si. In nanostructures such as $\mathrm{Si}$ nanowire, mean free path of phonon is restricted strongly by the interface or surface scattering. Thus phonon-drag effect is strongly reduced.

For the materials with low lattice thermal conductivity or metals, carrier contribution to the thermal conductivity becomes important. The electronic part to the thermal conductivity is calculated by considering the energy transfer of each electron under the condition $J=0$ in the Eq.(2). The energy carried by one electron may be considered to be $E$ - $E_{O V}$ where $E_{O V}$ is the average energy of electrons in the valley V. However, the correct energy is E-EF taking into account the recombination of excess carriers caused by the multiple carrier flow. Thus we can calculate the total energy transfer or thermal conductivity by multiplying $\left(E^{-} E_{F}\right) / e$ in the integral of Eq.(2) as given by

$$
\begin{aligned}
\kappa_{E} & =\frac{1}{4 \pi^{3} k T^{2}}\left[\sum_{V} \int \tau v_{X}^{2} f_{0}\left(1-f_{0}\right)\left(E(k)-E_{F}\right)^{2} d^{3} k-e^{2} S^{2} T^{2} \sum_{V} \tau v_{X}^{2} f_{0}\left(1-f_{0}\right) d^{3} k\right] \\
& =\sigma T\left[\frac{1}{e^{2} T^{2}} \frac{\sum_{V} \int \tau v_{x}^{2} f_{0}\left(1-f_{0}\right)\left(E-E_{F}\right)^{2} d^{3} k}{\sum_{V} \int \tau v_{x}^{2} f_{0}\left(1-f_{0}\right) d^{3} k}-S^{2}\right]
\end{aligned}
$$

IV-VI materials such as $\mathrm{PbTe}, \mathrm{PbSe}$, and $\mathrm{PbS}$ have small lattice thermal conductivity as low as $2 \mathrm{~W} / \mathrm{mK}$ at low temperature and relatively high carrier mobility. Thus the electronic thermal conductivity becomes important. 


\section{Seebeck Coefficients and Electronic Thermal}

\section{Conductivity of IV-VI Materials}

Figure 3 (a) shows the dependence of Seebeck coefficients on donor and acceptor concentrations for $\mathrm{PbS}, \mathrm{PbTe}$, and EuTe/PbTe superlattices at 300K, and Fig. 3(b) shows temperature dependence of Seebeck coefficient for PbTe and $\mathrm{PbS}$. The $\operatorname{dots} \mathbf{\square}, \bigcirc$, and $\square$ indicates the experimental data for $\mathrm{PbS}, \mathrm{PbTe}$, and $\mathrm{EuTe} / \mathrm{PbTe}$ superlattices, respectively, and solid, dashed, and dashed-dotted lines indicates theoretical dependence for $\mathrm{PbS}, \mathrm{PbTe}$, and $\mathrm{EuTe} / \mathrm{PbTe}$ superlattice, respectively. Detailed calculations and parameters for the $\mathrm{PbTe}$ and $\mathrm{EuTe} / \mathrm{PbTe}$ superlattice are given in Ref. 11. Band gaps and effective mass parameters for $\mathrm{PbS}$ and $\mathrm{PbTe}$ are indicated in table I.17,18) We used same effective masses for conduction and valence band of $\mathrm{PbS}$ owing to almost symmetric band edge structures at L-point. The Seebeck coefficients of the n-type and p-type PbTe films, and n-type EuTe/PbTe superlattices have been reported before.11,14) Enhancement of Seebeck coefficient in the EuTe/PbTe superlattices were obtained through the modulation of the density of states.14) The Seebeck coefficient of $\mathrm{p}$-type $\mathrm{PbTe}$ was higher than that of n-type owing to indirect valence band extrema near the L-point valence band tops. ${ }^{11)}$ Measurement system for the PbTe films and superlattices has been described in Ref.14. Experimental setup for the temperature dependence of Seebeck coefficient for $\mathrm{PbS}$ is shown in Fig.4. The sample film was contacted by pushing the sample on the $\mathrm{Cu}$ heat sink, and the temperatures of sample in both sides were 
measuresd by $\mathrm{Cu}$-Constantan thermocouples buried in the heat sink. Seebeck voltage was measured by measuring the voltage between $\mathrm{Cu}$ lines in high and low temperature sides.

Theoretical values for PbS films at $300 \mathrm{~K}$ agreed well with the experimental ones as well as $\mathrm{PbTe}$ and $\mathrm{EuTe} / \mathrm{PbTe}$ superlattice. Seebeck coefficient of the n-type $\mathrm{PbS}$ was higher than that of $\mathrm{PbTe}$, since $\mathrm{PbS}$ has large density of states compared to PbTe due to large effective masses. On the other hand, Seebeck coefficient of p-type $\mathrm{PbS}$ was comparable to that of $\mathrm{p}$-type $\mathrm{PbTe}$, and absolute value of the Seebeck coefficient for the p-type $\mathrm{PbS}$ was comparable to that of n-type $\mathrm{PbS}$ owing to the symmetric band structure with small higher band effect. In the temperature dependence of Seebeck coefficient shown in Fig.3(b), experimental values for the $\mathrm{n}$-type $\mathrm{PbS}$ agreed well with the theoretical values with relatively high Seebeck coefficient compared with theoretical values for $\mathrm{PbTe}$. The $\mathrm{PbTe}$ has been utilized as good thermoelectric materials with small thermal conductivity. However, Te is not abundant in the earth. Thus $\mathrm{PbS}$ is preferable if the comparable $\mathrm{ZT}$ is obtained for $\mathrm{PbS}$.

Lattice thermal conductivity of IV-VI materials such as $\mathrm{PbTe}, \mathrm{PbSe}$, and $\mathrm{PbS}$ has the value $2 \mathrm{~W} / \mathrm{mK}$ at room temperature, and the value decreases with the temperature. Electronic thermal conductivity becomes important for the IV-VI materials owing to the small lattice thermal conductivity, and the electronic thermal conductivity is calculated from Eq.(12). Figure 5(a) shows the calculated dependence of electronic thermal conductivity on temperature for $\mathrm{n}$-type $\mathrm{PbTe}$ with the parameter of carrier 
concentration. At room temperature, the electronic thermal conductivity is almost proportional to the carrier concentration as expected. In simple consideration, electronic thermal conductivity decreases with temperature owing to rapid decrease of carrier mobility. However, an enhancement of thermal conductivity with temperature is predicted by the theoretical calculation. This is considered to be due to a minority carrier effect. Minority carrier concentrations for the $\mathrm{PbTe}$ films with $\mathrm{N}_{\mathrm{D}}=1 \times 10^{19}, 5 \times 10^{18}$, $1 \times 10^{18}$, and $1 \times 10^{17} \mathrm{~cm}^{-3}$ are estimated to be $2.5 \times 10^{17}, 4.7 \times$ $10^{17}, 1.2 \times 10^{18}$, and $1.6 \times 10^{18} \mathrm{~cm}^{-3}$ at $800 \mathrm{~K}$, respectively. The enhancement of total carrier concentration is significant for low carrier concentration films. However, the lower thermal conductivity in higher carrier concentration film at high temperature in Fig.5(a) cannot be explained only by the carrier concentration enhancement. At low temperature, minority carrier concentration is negligibly small, and energy transfer or electronic thermal conduction occurs under the balance of thermal diffusion and drift currents, conducting relatively small energy. However, in two carrier conductions at high temperature, thermal diffusion current and drift current have the same direction for the minority carrier as shown in Fig.1, and balance between thermal diffusion current $\left(J_{e t}\right)$ and drift current $\left(J_{e d}\right)$ in the majority carrier is broken. Thus higher thermal flow is caused in the two carrier conduction because of the same directional flows of electrons and holes. The additional thermal flow is simply estimated to be $\left(E_{g}+3 k T\right)\left(J_{h d}+J_{\mathrm{ht}}\right) / e$ as the energy generated by the electron-hole recombinations, and the value becomes significant even if the minority carrier coincentration is 
relatively low, because the interband transition energy $\mathrm{E}_{\mathrm{g}}+3 k T$ is much higher than the intraband transition energy proportional to $k T$.

Enhancements in lattice thermal conductivity at high temperature have been reported for various materials.5) However, the enhancement of the thermal conductivity may be due to the electronic part derived here. Figure 5(b) shows comparison of electronic thermal conductivities for $\mathrm{n}$, $\mathrm{p}$-type $\mathrm{PbTe}$, and $\mathrm{n}$, $\mathrm{p}$-type $\mathrm{PbS}$ with carrier concentration of $1 \times$ $10^{19} \mathrm{~cm}^{-3}$. In this calculation, we assumed room temperature carrier mobilities $1500,750,600$, and $600 \mathrm{~cm}^{2} / \mathrm{Vs}$ for $\mathrm{n}$-type $\mathrm{PbTe}$, p-type $\mathrm{PbTe}$, n-type $\mathrm{PbS}$, and p-type $\mathrm{PbS}$, respectively, and temperature dependence $\mu(\mathrm{T}) \propto \mathrm{T}^{-2.5}$ for all the materials. ${ }^{19)}$ The theoretical value for $n$-type and $\mathrm{p}$-type $\mathrm{PbS}$ is the same because they have the same carrier mobility and effective masses. Among lead salt materials such as PbTe, $\mathrm{PbSe}$, and $\mathrm{PbS}$, enhancement of the electronic thermal conductivity is highest in $\mathrm{PbTe}$, because of temperature independent band gap above 400K, owing to direct gap to indirect gap transition,11) and smallest in $\mathrm{PbS}$ owing to positive temperature dependence of band gap with relatively large value and small carrier mobility. Thermoelectric figure of merits for n-type $\mathrm{PbTe}$ and $\mathrm{n}$-type $\mathrm{PbS}$ were calculated in the temperature range of $300-800 \mathrm{~K}$ assuming lattice thermal conductivity $\mathrm{K}_{\mathrm{L}} \propto \mathrm{T}^{-1.5}$. Figure 6 shows the calculated ZTs for the materials. Expected $\mathrm{ZT}$ for p-type $\mathrm{PbS}$ is the same with n-type $\mathrm{PbS}$ because Seebeck coefficients and carrier mobilities are almost same for n-type and p-type $\mathrm{PbS}$. $\mathrm{PbS}$ has smaller $\mathrm{ZT}$ than $\mathrm{PbTe}$ at room temperature owing to small carrier mobility. However, the ZT increases strongly 
with temperature, and it has comparable values with $\mathrm{PbTe}$ around 600-800K. Thus PbS is expected as good thermoelectric materials in this temperature range.

\section{Summary}

We described general calculations of Seebeck effect including metals, semiconductors, and quantum structures, which would be useful for both theoretical and experimental thermoelectric researchers. Mott formula is satisfied in simple metal approximation. The calculations for many valley semiconductors and nonparabolic cases were also described, and it was discussed how directional dependence appears in Seebeck coefficients. Theoretical calculations of Seebeck coefficient and electronic thermal conductivity were performed for IV-VI materials. Theoretical Seebeck coefficients of $\mathrm{p}^{-}$and $\mathrm{n}$-type $\mathrm{PbS}$ agreed well with experimental values as well as $\mathrm{PbTe}$ films and EuTe/PbTe superlattices. In IV-VI narrow gap materials, electronic part of thermal conductivity becomes important, and significant enhancement of thermal conductivity is caused by the minority carriers.

\section{Acknowledgements}

This work was financially supported by Suzuki foundation, and partly supported by Grants-in-Aid for Scientific Research (21360336) from the Japan Society for the Promotion of Science. 


\section{References}

1) T. C. Harman, P. J. Taylor, D. L. Spears and M. F. Walsh: J. Electron. Mater. 29, L1 (2000).

2) R. Venkatasubramanian, E. Siivoka, T. Colpitts and B. O’Quinn: Nature 413 (2001) 597.

3) A. L. Hochbaum, R. Chen, R. D. Delgado, W. Liang, E. C. Garnett, M. Najarian, A. Majumdar and P. Yang, Nature 451 (2008) 163.

4) I.Terasaki, Y. Sasago and K. Uchinokura: Phys. Rev. B 56 (1997) R 12685.

5) G. J. Snyder and E. S. Toberer, Nature materials 7, 105 (2008) 105.

6) M. Jonson and G. D. Mahan: Phys. Rev. B 21 (1980) 4223.

7) A. I. Boukai, Y. Bunimovich, J. T. Kheli, J. K. Yu, W. A. Goddard III and J. R. Heath: Nature 451 (2007) 168.

8) J. P. Heremans, V. Jovovic, E. S. Toberer, A. Saramat, K. Kurosaki, A. Charoenphakdee, S. Yamanaka and G. J. Snyder: Science 321 (2008) 554.

9) M. Asato, M. Ohkubo, T. Hoshino, F. Nakamura, N. Fujima and H. Tatsuoka: Materials Transactions, 49 (2008) 1760.

10) T. Hoshino, M. Asato, R.Zeller, and P. H. Dederichs: Phys. Rev. B 70 (2004) 094118.

11) A. Ishida, T. Yamada, D. Cao, Y. Inoue, M. Veis and T. Kita: J. Appl. Phys. 106 (2009) 023718.

12) T. H. Geballe and G. W. Hull: Phys. Rev. 98 (1955) 940.

13) J. M. Ziman: "Principles of the Theory of Solids" 2nd Edition, (Cambridge University Press, 1972).

14) A. Ishida, D. Cao, S. Morioka, M. Veis, Y. Inoue and T. Kita: Appl. Phys. Lett. 92 (2008) 182105.

15) J. P. Heremans, C. M. Thrush, D. T. Morelli: Phys. Rev. B 
$70(2004) 115334$.

16) A.Ishida, D. Cao, S. Morioka, Y. Inoue, and T. Kita: J. Electron. Mat. 38 (2009) 940.

17) H. Preier: Appl. Phys. 20 (1979) 189.

18) K. F. Cuff, M. R. Ellet, C. D. Kuglin and L. R. Williams:

Proc. 7th Int. Conf. Phys. Semicond. (Dunod, Paris 1964) pp.325.

19) Z. H. Dughaish: Physica B 322 (2002) 205. 
Table I Band parameters used for the theoretical calculations in $\mathrm{PbS} .{ }^{17,18)}$

\begin{tabular}{|c|c|c|c|c|c|}
\hline & $\begin{array}{c}\mathrm{E}_{\mathrm{g}}(\mathrm{T}) \\
{[\mathrm{meV}]}\end{array}$ & $\begin{array}{c}\mathrm{m}_{\mathrm{t}}(0) \\
{\left[\mathrm{m}_{0}\right]}\end{array}$ & $\begin{array}{c}\mathrm{m}_{1}(0) \\
{\left[\mathrm{m}_{0}\right]}\end{array}$ & $\begin{array}{c}\mathrm{m}_{\mathrm{t}}(\mathrm{T}) \\
{\left[\mathrm{m}_{0}\right]}\end{array}$ & $\begin{array}{c}\mathrm{m}_{1}(\mathrm{~T}) \\
{\left[\mathrm{m}_{0}\right]}\end{array}$ \\
\hline $\mathrm{PbS}$ & $263+\left(400+0.256 \mathrm{~T}^{2}\right)^{1 / 2}$ & 0.08 & 0.105 & $\frac{E_{g L}(T)}{E_{g L}(0)} m_{t}(0)$ & $\frac{E_{g L}(T)}{E_{g L}(0)} m_{l}(0)$ \\
\hline $\mathrm{PbTe}$ & $\begin{array}{c}167+\left(400+0.256 \mathrm{~T}^{2}\right) 1 / 2 \\
\text { Indirect gap: } 370 \\
\end{array}$ & 0.024 & 0.24 & $\frac{E_{g L}(T)}{E_{g L}(0)} m_{t}(0)$ & $\frac{E_{g L}(T)}{E_{g L}(0)} m_{l}(0)$ \\
& for T>400K & & & & \\
\hline
\end{tabular}




\section{Figure Captions}

Fig.1 Schematic diagram of electron and hole distribution on energy under temperature gradient. The $J_{e d}$ and $J_{h d}$ represent drift current densities for electrons and holes, respectively, and $J_{e t}$ and $J_{h t}$ represents thermal diffusion currents for electrons and holes, respectively.

Fig.2 Conduction band densities of states for bulk $\mathrm{Si}$, two-dimensional system grown to [111] direction with $10^{6}$ quantum wells/cm, and one-dimensional system with $10^{12}$ wires $/ \mathrm{cm}^{2}$.

Fig.3 (a) the dependence of Seebeck coefficient on donor and acceptor concentrations for $\mathrm{PbS}, \mathrm{PbTe},{ }^{11,14)}$ and $\mathrm{EuTe} / \mathrm{PbTe}$ superlattice ${ }^{14)}$ at $300 \mathrm{~K}$, and (b) the temperature dependence of Seebeck coefficient for $\mathrm{PbTe}$ and $\mathrm{PbS}$ with a carrier concentration of $1 \times 10^{19} \mathrm{~cm}^{-3}$. The $\operatorname{dots} \boldsymbol{\square}, \bigcirc$, and indicates the experimental data for $\mathrm{PbS}, \mathrm{PbTe}$, and EuTe/PbTe superlattices, respectively, and solid, dashed, and dashed-dotted lines indicates theoretical dependence for $\mathrm{PbS}, \mathrm{PbTe}$, and $\mathrm{EuTe} / \mathrm{PbTe}$ superlattice, respectively.

Fig.4 Seebeck voltage measurement system used to measure the temperature dependence of Seebeck coefficient of PbS.

Fig.5 (a) Dependence of electronic thermal conductivity on temperature for n-type $\mathrm{PbTe}$ with the parameter of carrier concentration, and (b) comparison of electronic thermal conductivities for $\mathrm{n}$, $\mathrm{p}$-type $\mathrm{PbTe}$, and $\mathrm{n}$, $\mathrm{p}$-type $\mathrm{PbS}$ with a 
carrier concentration of $1 \times 10^{19} \mathrm{~cm}^{-3}$.

Fig.6 Thermoelectric figure of merits for (a) n-type PbTe and (b) $\mathrm{n}$-type $\mathrm{PbS}$ in the temperature range of $300-800 \mathrm{~K}$ with $100 \mathrm{~K}$ step, calculated assuming temperature dependent lattice thermal conductivity $\mathrm{KL}_{\mathrm{L}} \propto \mathrm{T}^{-1.5}$ and carrier mobility $\mu \propto \mathrm{T}^{-2.5}$. 
Fig. 1
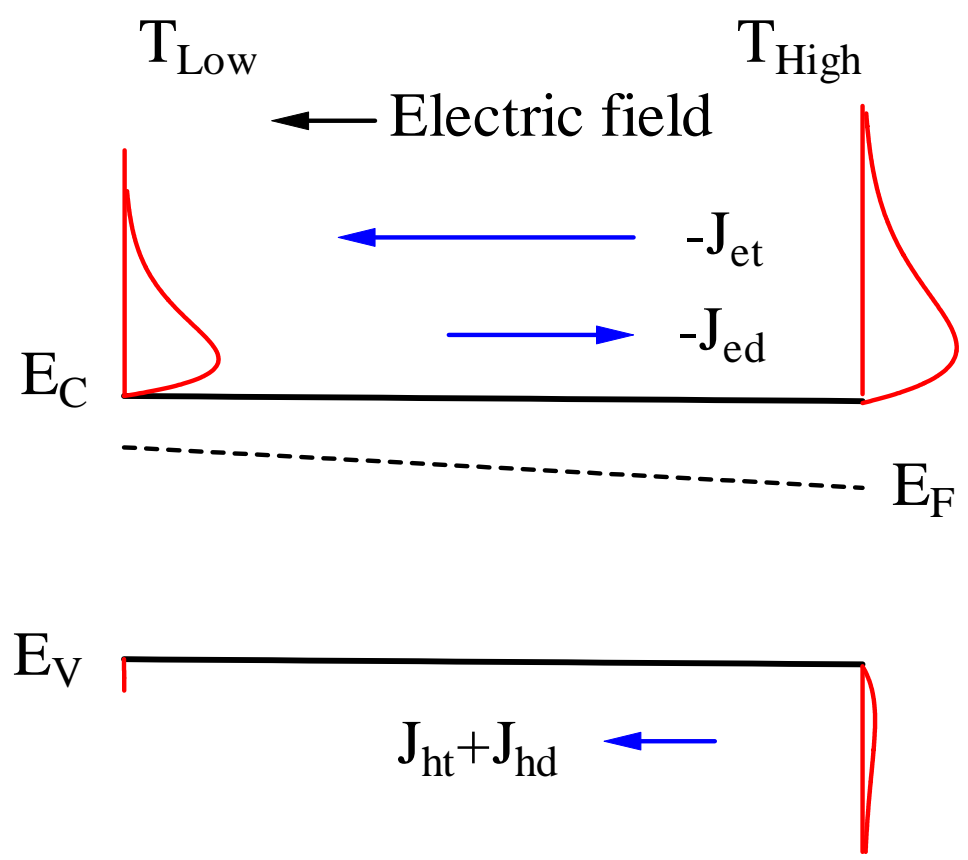
Fig.2

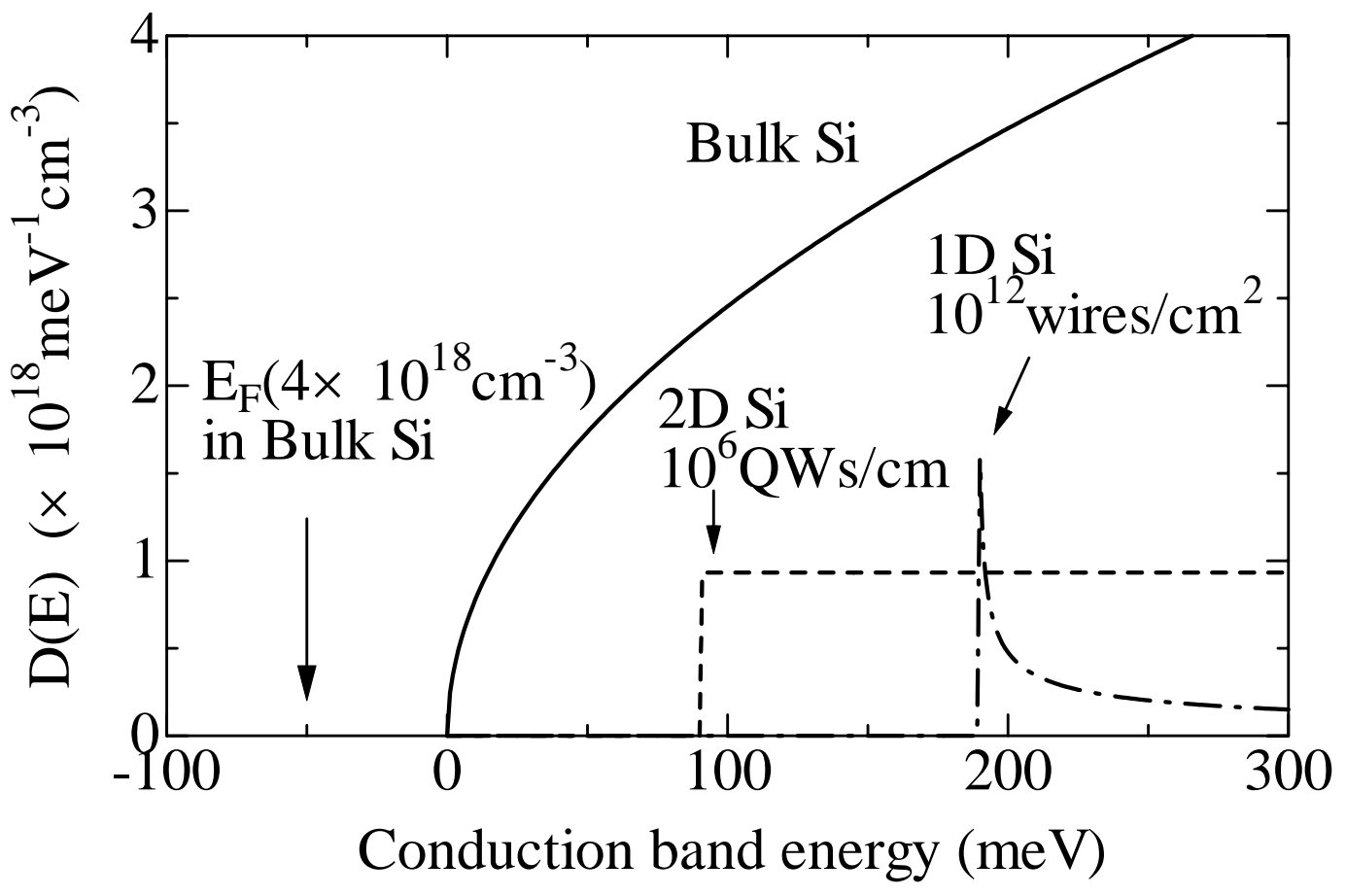


Fig. 3

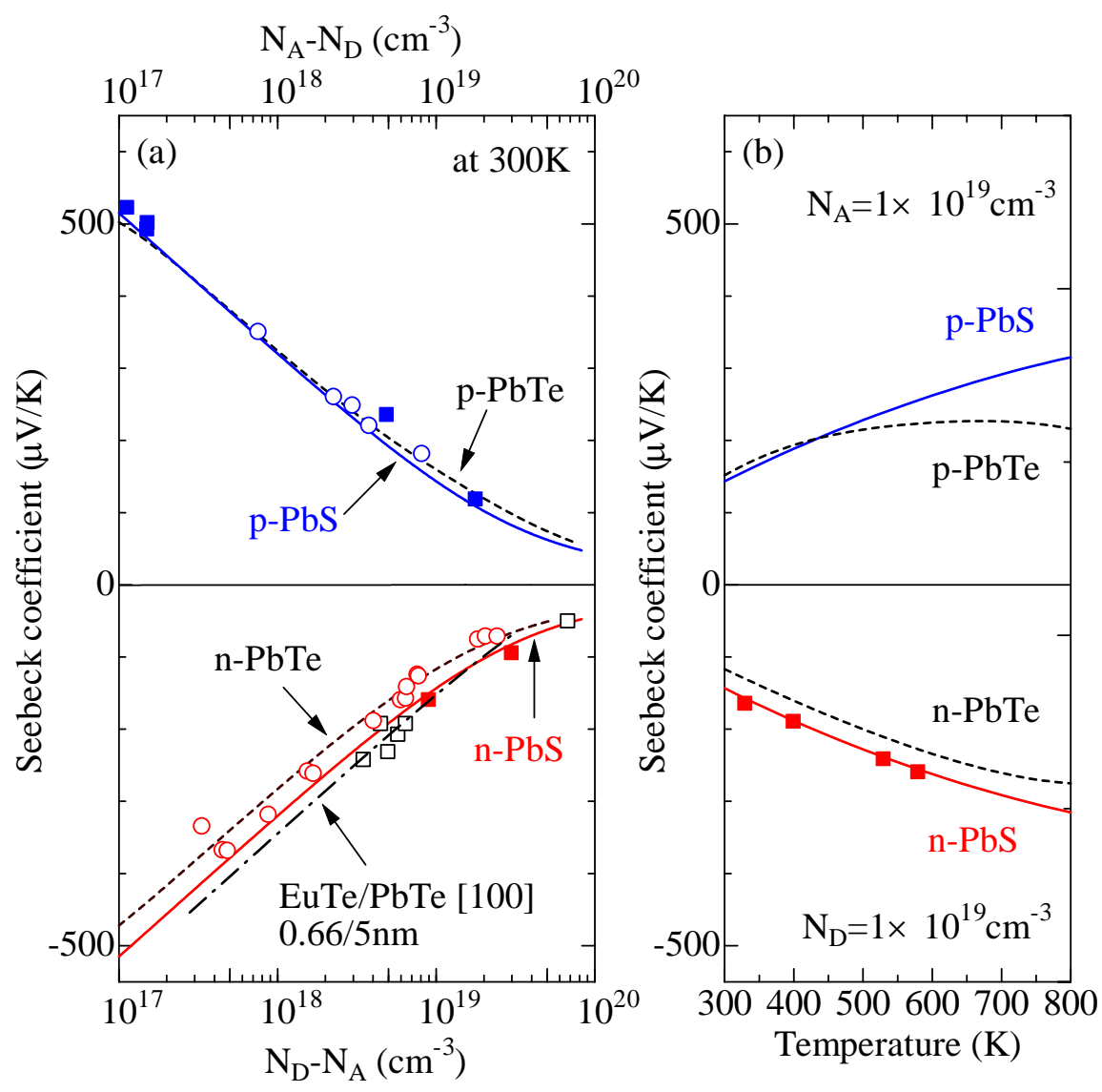


Fig. 4

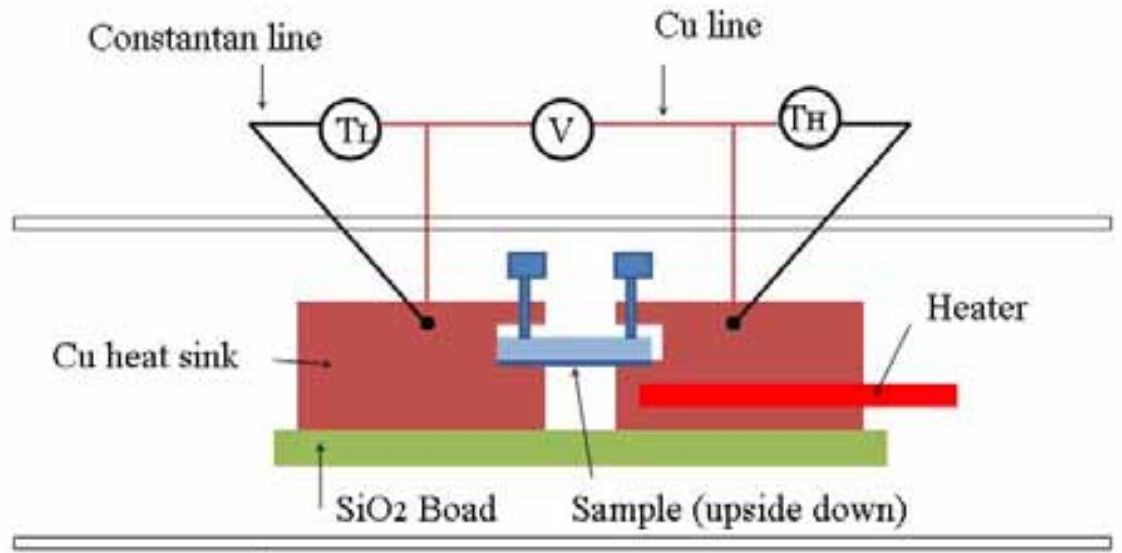

Temperature controlled furnace with $\mathrm{N} 2$ gas inside 
Fig. 5
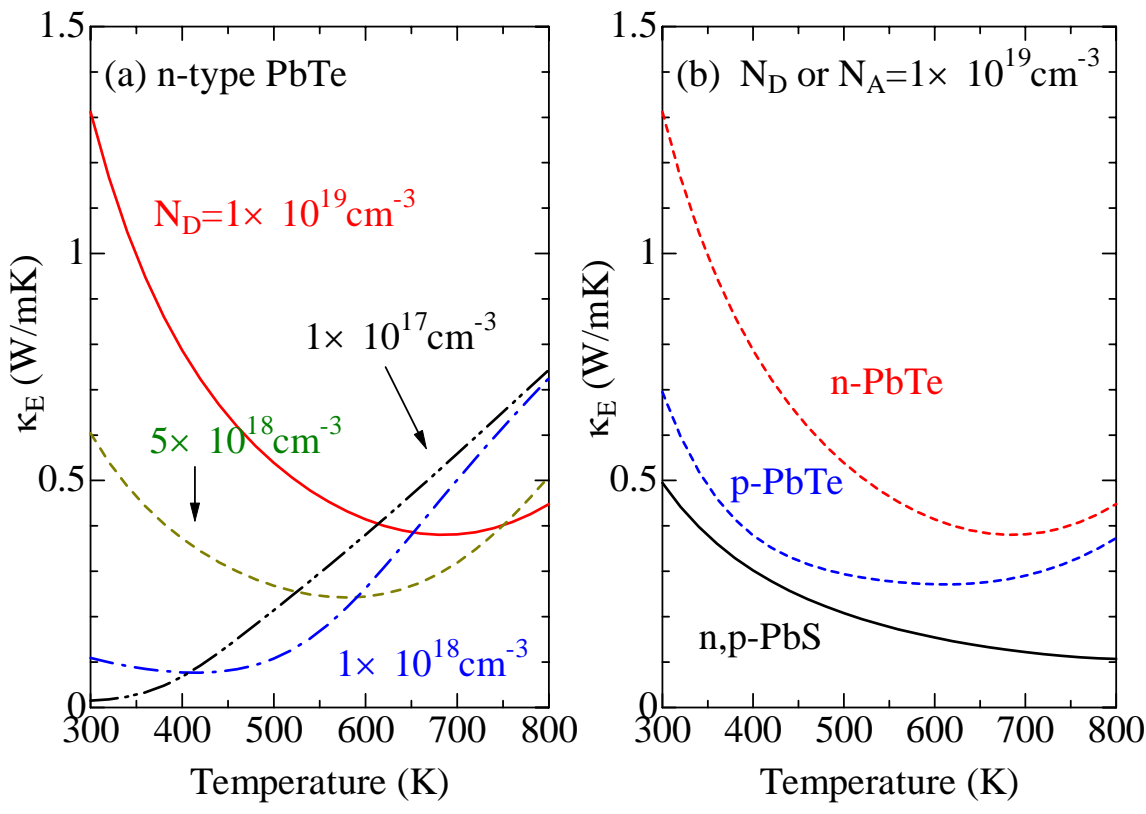
Fig. 6
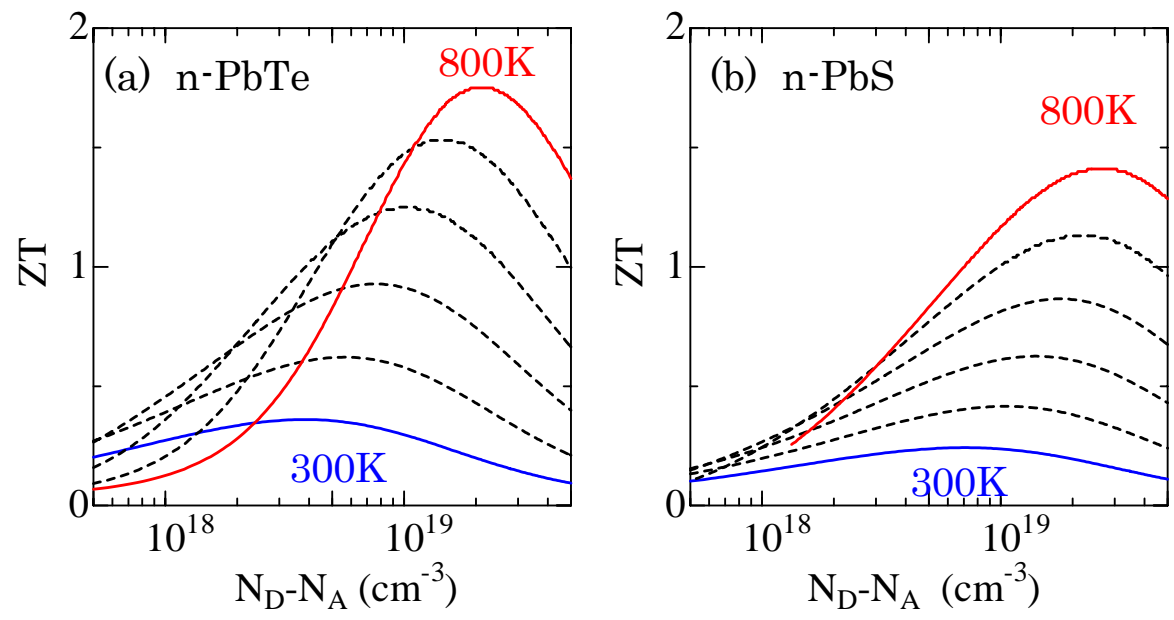